\title{
Downregulation of A disintegrin and metallopeptidase with thrombospondin motif type 1 by DNA hypermethylation in human gastric cancer
}

\author{
JING CHEN, CHUNDONG ZHANG, XIAOYANG XU, XINJIANG ZHU and DONGQIU DAI \\ Department of Gastrointestinal Surgery, The Fourth Affiliated Hospital of China Medical University, \\ Shenyang, Liaoning 110032, P.R. China
}

Received May 25, 2014; Accepted March 23, 2015

DOI: $10.3892 / \mathrm{mmr} .2015 .3667$

\begin{abstract}
A disintegrin and metallopeptidase with thrombospondin motif type 1 (ADAMTS1) is a metalloproteinase with antiangiogenic activity. It was previously observed that the mRNA and protein levels of ADAMTS1 are downregulated in primary gastric tumors. The aim of the present study was to examine whether the reduction in the expression of ADAMTS1 is due to aberrant methylation of the gene in primary gastric tumor tissues and gastric cancer cell lines. In addition, the association between ADAMTS1 methylation and clinicopathological features in were investigated in patients with primary gastric cancer. The results revealed that the frequency of ADAMTS1 methylation in primary gastric tumor tissues was significantly higher, compared with the corresponding normal gastric tissues. The relative mRNA expression levels of ADAMTS1 were significantly lower in the methylated primary gastric tumor tissues, compared with the unmethylated primary gastric tumor tissuess. A significant association was observed between the ADAMTS1 methylation status and the depth of tumor invasion and tumor, node, metastasis stage in primary gastric cancer. The mRNA expression of ADAMTS1 was significantly lower in $60 \%$ (3 of 5) of the gastric cancer cell lines. The relative mRNA expression levels of ADAMTS1 were significantly lower in the methylated gastric cancer cell lines, compared with the unmethylated gastric cancer cell lines. Furthermore, the expression of ADAMTS1 was significantly restored following treatment with the 5-Aza-2'-deoxycytidine demethylating agent in the MGC-803, HGC-27 and AGS gastric cancer cell lines, and the demethylation of the MGC-803 cell
\end{abstract}

Correspondence to: Professor Dongqiu Dai, Department of Gastrointestinal Surgery and Cancer Center, The Fourth Affiliated Hospital of China Medical University, 4 Chongshan East Street, Shenyang, Liaoning 110032, P.R. China

E-mail: daidq63@163.com

Key words: A disintegrin and metallopeptidase with thrombospondin motif type 1 , angiogenesis, methylation, gastric cancer line inhibited cell invasion. Together, these results suggested for the first time, to the best of our knowledge, ADAMTS1 as a novel antitumor protease, and this function was lost following epigenetic silencing in the gastric cancer cells and gastric tumor tissues. Therefore, the aberrant methylation of ADAMTS1 may be involved in the development and progression of gastric cancer.

\section{Introduction}

A disintegrin and metallopeptidase with thrombospondin motifs (ADAMTS) is a matrix metalloproteinase, which consists of multiple domains, including propeptide, metalloproteinase, disintegrin, spacer region domains, and domains containing thrombospondin type I (TSP-I) motifs (1). The ADAMTS family contains 19 members. ADAMTS are characterized by the presence of additional TSP-I motifs at their C-terminal region, while epidermal growth factor-like, transmembrane and cytoplasmic domains are missing $(2,3)$. Several of these genes are involved in a variety of human diseases, including connective tissue disorder, inflammation, arthritis, cancer and thrombotic thrombocytopenic purport (4-10). Certain ADAMTS are involved in the progression of cancer, including cell proliferation, apoptosis, migration, invasion and angiogenesis (11). Although the precise role of ADAMTS in the development and progression of cancer remains to be elucidated, previous studies have provided evidence of dysregulation of certain ADAMTS in different types of cancer.

ADAMTS1, the first described member of the ADAMTS family, is widely expressed in various human tissues $(1,12)$. Previous studies have demonstrated that ADAMTS1 can degrade aggrecan and versican, which are components of extracellular matrix (ECM) barriers $(13,14)$. It is also considered to be a potent anti-angiogenic factor, which inhibits endothelial cell proliferation and angiogenesis $(15,16)$. ADAMTS1 inhibits angiogenesis through binding to vascular endothelial growth factor (VEGF) 165 and inhibiting VEGF-A165-stimulated phosphorylation of VEGFR-2 (17). In addition, it has decreased expression levels in a number of types of cancer $(8,18-20)$, however, the underlying mechanism remains to be elucidated. 
Gastric cancer is considered to be caused by multiple factorS and develop in a multistep process. Epigenetics, particularly DNA methylation, has been investigated as one of the causes of the progression and development of gastric cancer (21). Several studies have indicated that DNA hypermethylation is an important mechanism in the transcriptional silencing of tumor-associated genes in gastric cancer. Our previous study demonstrated that decreased expression of ADAMTS1 is observed in primary gastric cancer (22). This suggests the possibility that the loss of ADAMTS1 function may be an important factor in tumor angiogenesis. To further examine the relevance of ADAMTS1 as a putative tumor-suppressor enzyme, the present study aimed to analyze the epigenetic mechanisms controlling its expression in gastric cancer cells and tissues. To the best of our knowledge, no previous studies have investigated whether the abnormal gene expression of ADAMTS1 in primary gastric cancer is due to epigenetic alterations in the pattern of DNA methylation. The present study aimed to examine the expression of ADAMTS1 in gastric cancer and its association with aberrant methylation.

\section{Materials and methods}

Tissues samples. Paired normal and tumor samples were obtained from 56 patients with gastric cancer, who underwent curative resection between March 2009 and October 2009, at The Fourth Affiliated Hospital of China Medical University. None of these patients had undergone chemotherapy or radiotherapy prior to surgery. The patient group consisted of 41 males $(73.2 \%)$ and 15 females (26.8\%) with a mean age of 59.8 years (range, 30-89 years). All tissues were frozen in liquid nitrogen immediately following surgery and stored at $-80^{\circ} \mathrm{C}$ until genomic DNA preparation. The present study was approved by the ethics committee of the Fourth Affiliated Hospital of China Medical University (Shenyang, China), and written informed consent was obtained from all patients.

Cell lines. The SGC-7901, BGC-823, MGC-803, HGC-27 and AGS human gastric cancer cell lines and GES-1 immortalized normal gastric cell line were cultured in RPMI-1640 (Gibco Life Technologies, Carlsbad, CA, USA), supplemented with $10 \%$ fetal bovine serum (Gibco Life Technologies), penicillin $(100 \mathrm{IU} / \mathrm{ml})$ and streptomycin $(100 \mu \mathrm{g} / \mathrm{ml})$ (Sangon Biotech Co., Ltd., Shanghai, China), and incubated in a humidified incubator containing $5 \% \mathrm{CO}_{2}$ at $37^{\circ} \mathrm{C}$. The SGC-7901, BGC-823, MGC-803, HGC-27 and AGS cells were obtained from the Institute of Biochemistry and Cell Biology, Chinese Academy of Sciences (Shanghai, China). The GES-1 cell was obtained from the Oncology Institute of China Medical University (Shenyang, China).

$R N A$ isolation and reverse-transcription-quantitative polymerase chain reaction ( $R T-q P C R)$. The total RNA from the cultured cells (density, $5 \times 10^{6}$ ) were isolated using TRIzol reagent (Invitrogen Life Technologies, Carlsbad, CA, USA). The DNase I-treated $\left(1 \mu \mathrm{l} ; 37^{\circ} \mathrm{C}\right.$ for $45 \mathrm{~min}$; Fermentas, Vilnius, Lithuania) total RNA ( $2 \mu \mathrm{g})$ was converted to cDNA using the RevertAid First Strand cDNA Synthesis kit (Fermentas).
The reverse transcriptase reaction was, as described previously (23). The primers for the human ADAMTS1 gene were forward 5'-AAGCTGCTGATGGCACATATATTCA-3' and reverse 5'-TTTTAGGTCGAAGGGCATTGC-3', which generated a 195 base pair (bp) fragment. GAPDH was also amplified as an internal control using the following primers: forward 5'-GAAGGTCGGAGTCAACGGAT-3' and reverse 5'-CCTGGAAGATGGTGATGGGAT-3', which generated a 224 bp fragment. All primers were obtained from Sangon Biotech Co., Ltd. The qPCR was performed with the PTC-100 cyling machine (MJ Research, Inc., Waltham, MA, USA) in a volume of $50 \mu \mathrm{l}$, containing 10X DreamTaq buffer (Fermentas), $2 \mathrm{mM}$ dNTP Mix, $0.4 \mu \mathrm{M}$ each primer and 1.25 units DreamTaq. Initial denaturation was set at $95^{\circ} \mathrm{C}$ for $5 \mathrm{~min}$, followed by 35 cycles of denaturation at $95^{\circ} \mathrm{C}$ for $20 \mathrm{sec}$, an annealing step at $58^{\circ} \mathrm{C}$ for $20 \mathrm{sec}$ and an extension step at $72^{\circ} \mathrm{C}$ for $30 \mathrm{sec}$. A final extension step at $72^{\circ} \mathrm{C}$ for $5 \mathrm{~min}$ was performed. The qPCR products were separated on $1.5 \%$ agarose gels and quantified using a Fluor Chem 2.0 system (Bio-Rad Laboratories, Inc., Hercules, CA, USA). The mRNA levels were determined by quantifying the intensities of the qPCR products against GAPDH. Low and high mRNA levels were classified by the estimated value below and above the median value.

Genomic DNA isolation and methylation-specific PCR $(M S P)$. The genomic DNA was prepared from the cell lines and fresh tissues using a phenol/chloroform procedure and was modified using bisulfite, as described previously (24). The DNA $(2 \mu \mathrm{g})$ was purified using a Wizard DNA Clean-Up system (Promega Corporation, Madison, USA), precipitated with $100 \%$ ethanol (Sangon Biotech Co., Ltd.) and resuspended in $30 \mu 1$ Tris-EDTA buffer (Sigma-Aldrich, St. Louis, MO, USA). The aliquot $(2 \mu \mathrm{l})$ was used as a template and $2 \mu \mathrm{g}$ DNA from each sample was treated with sodium bisulfite (Sigma-Aldrich) at $50^{\circ} \mathrm{C}$ for $16 \mathrm{~h}$. The DNA was then washed with $70 \%$ ethanol and dissolved in $15 \mu 1 \mathrm{TE}$. The methylated primer sequences were: Forward 5'-CGTTAGGTATTAATTTTCGC-3' and reverse 5'-CGCTACAATTCTACCGACGCG-3' (151 bp); the unmethylated primer was: Forward 5'-TGTTAGGTATTAATTTTTGT-3' and reverse 5'-CACTACAATTCTACCAACACA-3' (151 bp) (25). The reaction mixture contained $2 \mu \mathrm{l}$ DNA in a volume of $50 \mu \mathrm{l}$, containing $2 \mu \mathrm{l}$ of each primer, 10X DreamTaq buffer, $2.0 \mathrm{mM}$ dNTP mix and 1.25 units DreamTaq (Fermentas.) The complete MSP conditions were as follows: $94^{\circ} \mathrm{C}$ for $5 \mathrm{~min}, 94^{\circ} \mathrm{C}$ for $30 \mathrm{sec}, 40$ cycles of $72^{\circ} \mathrm{C}$ for $45 \mathrm{sec}, 56^{\circ} \mathrm{C}$ for $30 \mathrm{sec}, 72^{\circ} \mathrm{C}$ for $30 \mathrm{sec}$ and $72^{\circ} \mathrm{C}$ for $10 \mathrm{~min}$. Human placental DNA, treated in vitro with Sss I methylase via MSP (New England Biolabs, Inc. Ipswich, MA, USA), served as a positive control for the methylated reaction. Water was used as a negative PCR control. The MSP products were separated using electrophoresis through a $2.5 \%$ agarose gel containing ethidium bromide, and were quantified using a Fluor Chem 2.0 system. Each MSP was repeated at least once to confirm the results.

Treatment of cells with 5-Aza-2'-deoxycytidine (5-Aza-dC). The six cell lines (density, $5 \times 10^{6}$ ) were incubated in culture media (RPMI-1640) at room temperature with $5 \mu \mathrm{M}$ 5-Aza-dC 
Table I. Methylation of A disintegrin and metallopeptidase with thrombospondin motif type 1 in primary gastric tumor tissues and its association with clinicopathological factors.

\begin{tabular}{|c|c|c|c|c|}
\hline Clinicopathological factor & $\begin{array}{c}\text { Number of } \\
\text { patients }\end{array}$ & Methylated (\%) & Unmethylated (\%) & P-value \\
\hline Gender & & & & 0.435 \\
\hline Male & 41 & $19(46.3)$ & $22(53.7)$ & \\
\hline Female & 15 & $8(53.3)$ & $7(46.7)$ & \\
\hline Age (years) & & & & 0.540 \\
\hline$\geq 65$ & 18 & $9(50)$ & $9(50)$ & \\
\hline$<65$ & 38 & $18(47.4)$ & $20(52.6)$ & \\
\hline Tumor size $(\mathrm{cm})$ & & & & 0.124 \\
\hline$\geq 3$ & 34 & $19(55.9)$ & $15(44.1)$ & \\
\hline$<3$ & 22 & $8(36.4)$ & $14(63.6)$ & \\
\hline Differentiation & & & & 0.297 \\
\hline Good/moderate & 28 & $12(42.9)$ & $16(57.1)$ & \\
\hline Poor & 28 & $15(53.6)$ & $13(46.4)$ & \\
\hline Depth of tumor invasion & & & & 0.048 \\
\hline pT1-pT2 & 15 & $4(26.7)$ & $11(73.3)$ & \\
\hline pT3-pT4 & 41 & $23(56.1)$ & $18(43.9)$ & \\
\hline TNM stage & & & & 0.015 \\
\hline I-II & 17 & $4(23.5)$ & $13(76.5)$ & \\
\hline III-IV & 39 & $23(59.0)$ & $16(41.0)$ & \\
\hline Lymph node metastasis & & & & 0.165 \\
\hline Positive & 42 & $18(42.8)$ & $24(57.2)$ & \\
\hline Negative & 14 & $9(64.3)$ & $5(35.7)$ & \\
\hline
\end{tabular}

TNM, tumor, node, metastasis.

demethylating agent (Sigma-Aldrich) for 3 days. Furthermore, the MGC-803 cells were incubated in culture media with 2.5 , 5 or $10 \mu \mathrm{M}$ of 5 -Aza-dC for 3 days, with daily media replacement. These cells were then analyzed using RT-PCR, with the MGC-803 cells also analyzed using immunofuorescence on the third day.

Immunofluorescence. Indirect immunofluorescence was performed on the ADAMTS1 cells grown in 24-well plates (Costar Corporation, Corning, NY, USA). The MGC-803 cells were fixed in $4 \%$ paraformaldehyde (Sangon Biotech Co., Ltd.) at $25^{\circ} \mathrm{C}$ for 15 min, permeabilized with $0.2 \%$ Triton X-100 (Sigma-Aldrich) and then washed three times with phosphate-buffered saline (PBS). The cells were incubated with rabbit anti-human ADAMTS1 polyclonal antibody (1:400; ab39194; Abcam, Cambridge, UK) overnight at $4^{\circ} \mathrm{C}$, followed by incubation in the dark at room temperature with a 1:100 dilution of goat anti-rabbit rhodamine-conjugated immunoblobulin G-R (sc-2091; Santa Cruz Biotechnology, Inc., Santa Cruz, CA, USA) for $2 \mathrm{~h}$. The nuclei were visualized using Hoechst 33258 counterstaining (Sangon Biotech Co., Ltd.) and examined using a fluorescence microscope (Olympus BX-40; Olympus, Tokyp, Japan).

Matrigel invasion assay. An invasion assay was performed using an $8 \mu \mathrm{m}$ pore size membrane coated with Matrigel
(Corning Life Sciences). The MGC-803 cells $\left(2 \times 10^{4}\right)$ in $200 \mu \mathrm{l}$ serum-free 1640 media were seeded onto Matrigel-coated Transwell filters (Corning Life Sciences) in the upper chambers. The lower chambers contained $500 \mu 1$ RPMI-1640 medium (Invitrogen Life Technologies) with $10 \%$ FBS to serve as a chemoattractant, and the chambers were incubated at $37^{\circ} \mathrm{C}$ in a humidified $5 \% \mathrm{CO}_{2}$. After $24 \mathrm{~h}$, the non-invading cells on the upper membrane surface were removed using cotton swabs. The cells on the lower side of the membrane (invading cells) were fixed in 4\% paraformaldehyde for $30 \mathrm{~min}$ and then stained with methylrosanilinium chloride (Sangon Biotech Co., Ltd.). The invaded cells were viewed under the Olympus BX-40 microscope and counted in five randomly selected fields (magnification, x200). The invasion abilities of the cancer cells were expressed as the mean number of cells in the five fields. The assay was performed in three independent experiments.

Data analysis. Statistical analysis was performed using the SPSS statistical software package, version 17.0 (SPSS, Inc., Chicago, IL, USA). The $\chi^{2}$ test was used to determine the association between ADAMTS1 methylation and the clinicopathological features (Table I). In addition, the non-parametric Mann-Whitney U test was used to determine the relative mRNA levels of ADAMTS1 in the cell lines, and their association with 
A

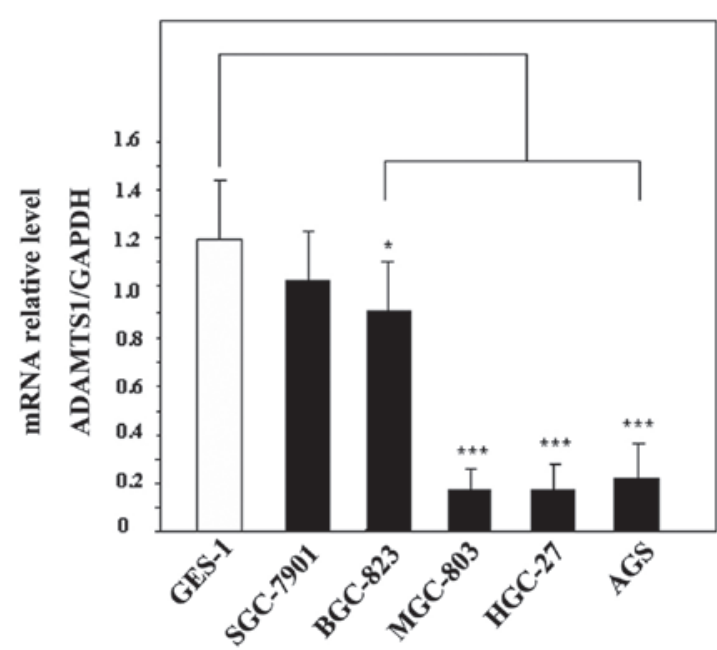

B

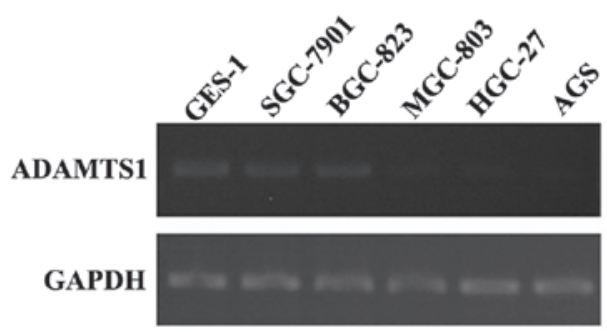

Figure 1. mRNA expression levels of ADAMTS1 in six gastric cell lines. (A) mRNA eof ADAMTS1 in the GES-1 normal gastric cell line and five gastric cancer cell lines. GAPDH was used as a control. (B) Relative mRNA expression levels of ADAMTS1 in the GES-1 normal gastric cell line and five gastric cancer cell lines. (Mann-Whitney U test; ${ }^{*} \mathrm{P}<0.05$ and ${ }^{* * *} \mathrm{P}<0.001$, compared with the GES-1 cell line). Data are presented as the mean \pm standard deviation. ADAMTS1, A disintegrin and metallopeptidase with thrombospondin motif type 1.

methylation. $\mathrm{P}<0.05$ was considered to indicate a statistically significant difference.

\section{Results}

mRNA expression of ADAMTS1 in cell lines. Representative examples are shown in Fig. 1A. The expression of ADAMTS1 was undetectable in the MGC-803, HGC-27 and AGS gastric cancer cell lines of the six cell lines examined. Statistically, the mRNA expression of ADAMTS1 was significantly lower in the BGC-823, MGC-803, HGC-27 and AGS gastric cancer cell lines, compared with the GES-1 normal gastric cell line. $(\mathrm{P}=0.022, \mathrm{P}=0.000, \mathrm{P}=0.000$ and $\mathrm{P}=0.000$, respectively; Fig. 1B).

Aberrant methylation of ADAMTS1 in the cell lines. The status of DNA methylation in the six cell lines was examined. Methylation was observed in the MGC-803, HGC-27 and AGS, gastric cancer cell lines, whereas no methylation was observed in the SGC-7901 and BGC-823 gastric cancer cell lines or the GES-1 normal gastric cell line (Fig. 2A). The statistical analyses demonstrated that the relative mRNA levels of ADAMTS1 were significantly lower in the methylated gastric cancer cell lines, compared with the unmethylated gastric cancer cell lines $(\mathrm{P}=0.036$; Fig. $2 \mathrm{~B})$ Furthermore, the promoter methylation sequence of ADAMTS1 was detected following treatment with sodium bisulfate in the methylated MGC-803 cell line, and all the CG bases remained as CG bases, which confirmed that the ADAMTS1 promoter was hypermethylated in the MGC-803 cell line (Fig. 2C).

mRNA and protein expression levels of ADAMTS1 in cell lines following treatment with 5-Aza- $d C$. In the present study, the six cell lines were treated with $5-\mathrm{Aza}-\mathrm{dC}$, which resulted in restoration of the mRNA expression of ADAMTS1 in the MGC-803, HGC-27 and AGS cell lines, determined using RT-qPCR analysis (Fig. 3A). As shown in Fig. 3B, the relative mRNA levels of ADAMTS1 were significantly higher following treatment with 5-Aza-dC in the MGC-803, HGC-27 and AGS cell lines, compared with those prior to treatment with 5-Aza-dC $(\mathrm{P}<0.001)$. The MGC-803 cell line was then treated for 3 days with $2.5,5$ or $10 \mu \mathrm{M} 5$-Aza-dC. The results revealed that the mRNA expression of ADAMTS1 was significantly upregulated following 5-Aza-dC treatment (Fig. 4A). In addition, the effect of 5-Aza-dC on the expression of ADAMTS1 was dose-dependent, with higher doses resulting in increased changes in the expression of ADAMTS1 (Fig. 4B). The present study also examined the protein expression of ADAMTS1 using immunofluorescence, and observed a marked upregulation in ADAMTS1 (Fig. 4C).

Inhibition of MGC-803 cell invasion following treatment with 5-Aza-dC. The invasive properties of the MGC-803 were affected by treatment with $10 \mu \mathrm{M} 5$-Aza-dC for 3 days. The results demonstrated that the number of invading MGC-803 cells was significantly reduced following 5-Aza-dC treatment, compared with the control group $(51 \pm 8.47$ vs. $206 \pm 18.11$; $\mathrm{P}<0.001$; Fig. 4D and E).

ADAMTS1 methylation in gastric cancer and clinicopathologic correlations. Our previous study reported low mRNA expression levels of ADAMTS1 in 56 primary gastric tumors. In the present study, the methylation status of the ADAMTS1 promoter was assessed in these primary gastric tumor tissues and in normal gastric tissues using MSP. Representative examples of the MSP analysis are shown in Fig. 5A. Methylation of the ADAMTS1 gene was detected in $27(48 \%)$ primary gastric tumor tissues and eight (14\%) corresponding normal gastric tissues. Methylation of the ADAMTS1 gene was significant higher in primary gastric tumor tissues compared with normal gastric tissues $(\mathrm{P}=0.009)$. In addition the relative mRNA levels of ADAMTS1 were significantly lower in the methylated primary gastric tumor tissues compared with those in the unmethylated primary gastric tumor tissues $(\mathrm{P}=0.043$; Fig. $5 \mathrm{~B})$. The present study also investigated the association between ADAMTS1 methylation and the clinicopathological features of the patients, 
A

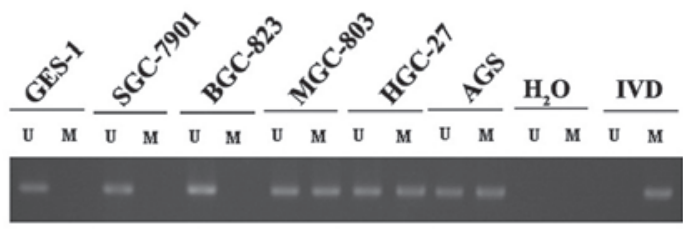

B

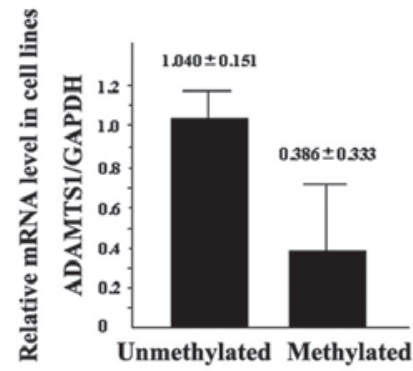

C

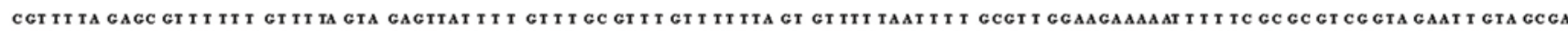

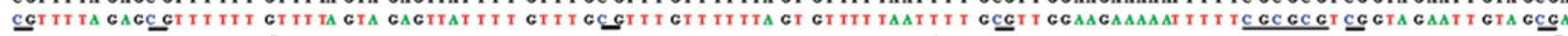
(n)

Figure 2. Methylation analysis of ADAMTS1 in the six gastric cell lines. (A) Methylation status of ADAMTS1 in the GES-1 normal gastric cell line and five gastric cancer cell lines. (B) Relative mRNA levels of ADAMTS1 in the unmethylated gastric cell lines and methylated gastric cell lines (Mann-Whitney $\mathrm{U}$ test; $\mathrm{P}=0.036)$. (C) Bisulphite sequence electropherogram of methylated MGC-803. CG, which remained unchanged are underlined in black. Data are presented as the mean \pm standard deviation. U, unmethylated; M, methylated; IVD, positive control; $\mathrm{H}_{2} \mathrm{O}$, negative control; ADAMTS1, A disintegrin and metallopeptidase with thrombospondin motif type 1 .

A

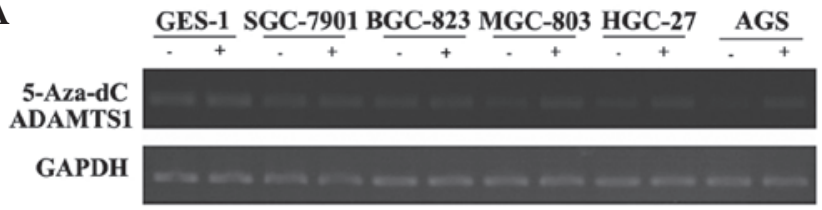

B

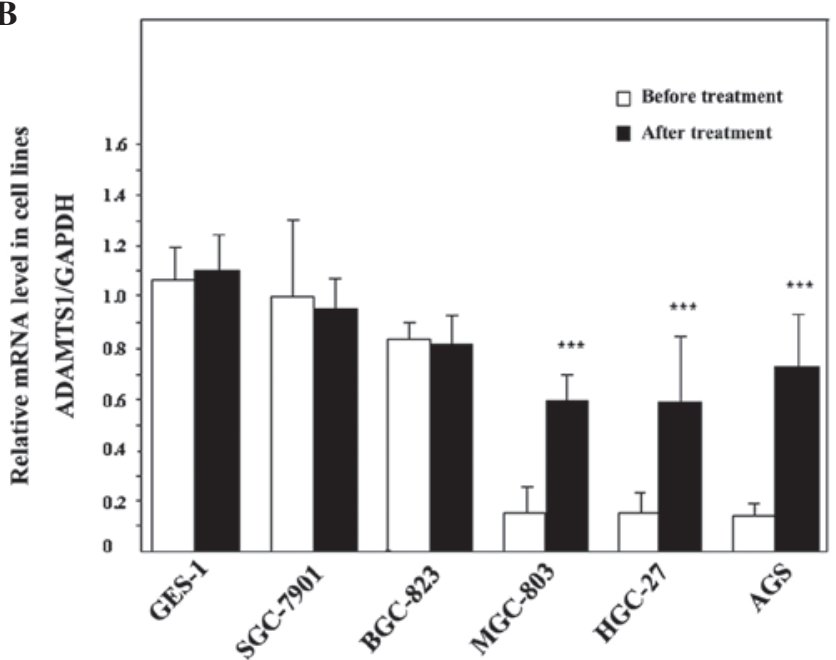

Figure 3. mRNA expression levels of ADAMTS1 in gastric cell lines before and after treatment with 5-Aza-dC. (A) mRNA expression levels of ADAMTS1 in the GES-1 normal gastric cell line and five gastric cancer cell lines were analyzed using reverse transcription-quantitative polymerase chain reaction analysis. (B) Relative mRNA expression levels of ADAMTS1 in the GES-1 normal gastric cell line and five gastric cancer cell lines (Mann-Whitney $\mathrm{U}$ test; ${ }^{* * *} \mathrm{P}<0.001$, vs. before treatment). Data are presented as the mean \pm standard deviation. ADAMTS1, A disintegrin and metallopeptidase with thrombospondin motif type 1; 5-Aza-dC, 5-Aza-2'-deoxycytidine.

including age, gender, size, differentiation, depth of tumor invasion, tumor, node, metastasis (TNM) Stage and lymph node metastasis. A significant association was observed between the ADAMTS1 methylation status, depth of tumor invasion and TNM stage. Methylation was significantly more frequent in primary tumor tissues at the pT3-pT4 stages, compared with those with at the pT1-pT2 stages $(\mathrm{P}=0.048)$. Methylation was significantly more frequent in primary tumor tissues at the III-IV stage, compared with those with at the I-II stages $(\mathrm{P}=0.015)$. None of the other clinicopathological factors were significantly associated with ADAMTS1 methylation (Table I).

\section{Discussion}

The present study investigated the mRNA expression of ADAMTS1 in five human gastric cancer cell lines and one immortalized normal gastric cell line, and analyzed the methylation status of ADAMTS1 using MSP in the cell lines and primary gastric tumor tissues. Furthermore, the present study also examined whether the loss of the expression of ADAMTS1 ws due to aberrant methylation of the gene. To the best of our knowledge, the present study is the first concerning the expression methylation status of ADAMTS1 in human gastric cancer.

Although the functional roles of ADAMTS1 in carcinogenesis remain to be fully elucidated, the anti-angiogenic properties of ADAMTS1 have been described. A previous report suggested that ADAMTS1 gene transfer inhibited angiogenesis in vitro and in vivo, possibly as a result of the induction of endothelial cell apoptosis by ADAMTS1, which occurs independently of protease activity (16). It sequesters the potent angiogenic stimulant, VEGF, from interacting with its receptors, preventing an angiogenic effect. ADAMTS1 also cleaves large TSP1, and cleaved TSP-1 demonstrates anti-angiogenic activity (26). Several studies have also demonstrated that ADAMTS1 is downregulated in breast 
A

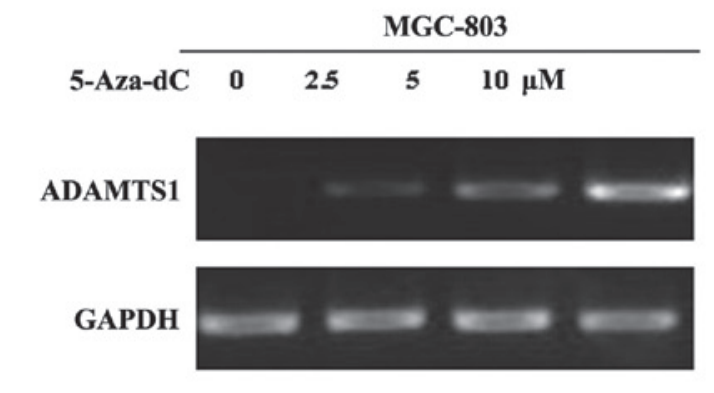

C

MGC-803

5-Aza-dC $\mathbf{0}$

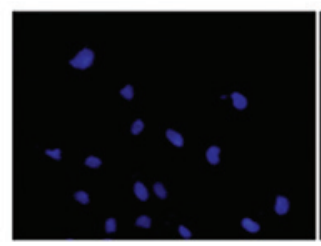

2.5

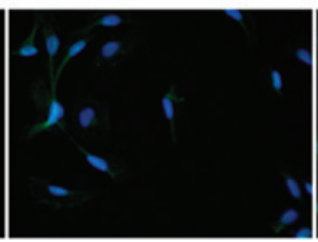

$\mathbf{B}$

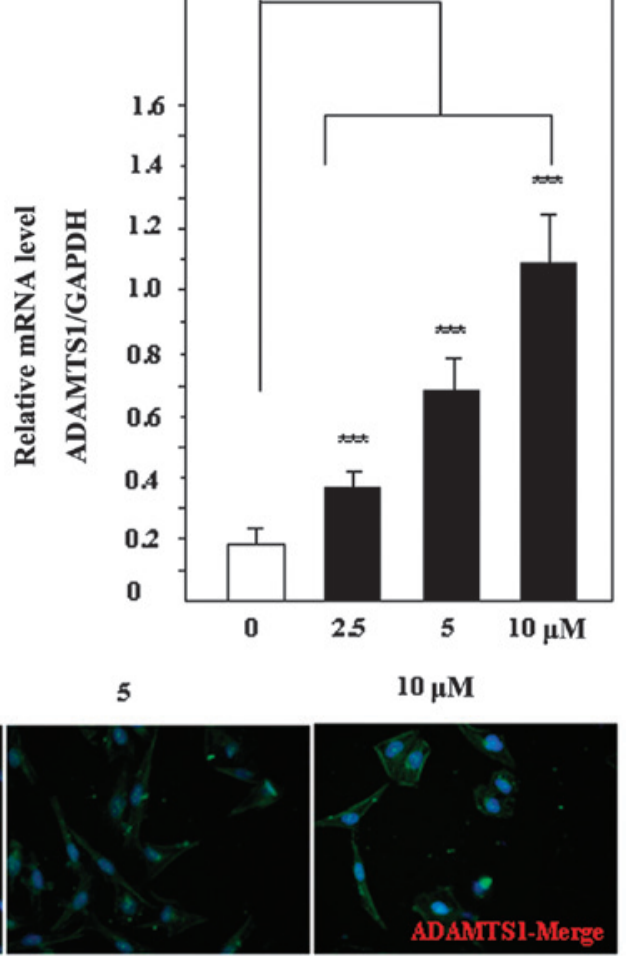

D
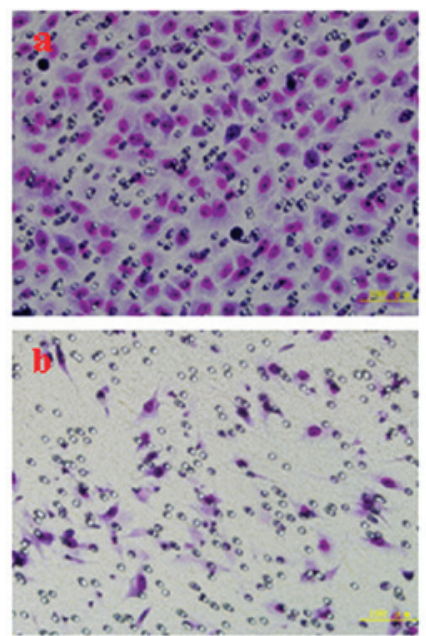

$\mathbf{E}$

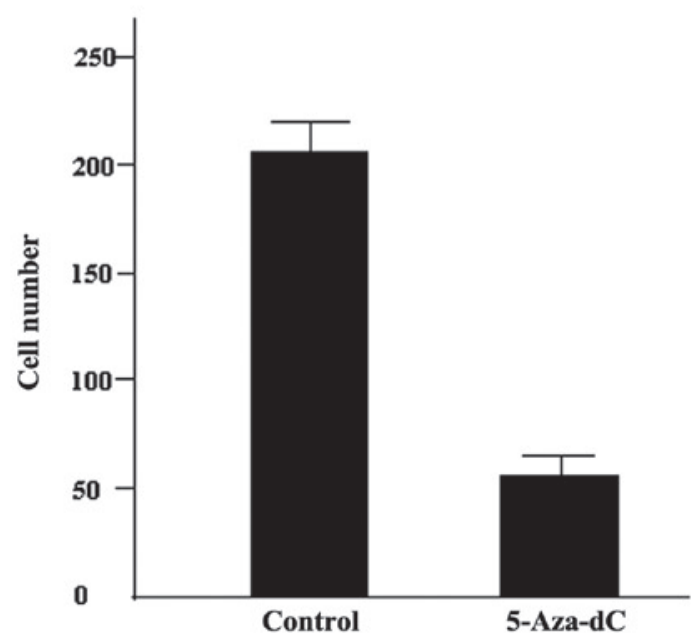

Figure 4. mRNA and protein expression levels of ADAMTS1 in the MGC-803 cell line prior to and following treatment with different concentrations of 5-Aza-dC for 3 days. (A) mRNA expression of ADAMTS1 prior to and following treatment with different concentrations of 5-Aza-dC using reverse transcription-quantitative polymerase chain reaction analysis. (B) ADAMTS1 relative mRNA levels prior to and following treatment with different concentrations of 5-Aza-dC (Mann-Whitney U test; $\left.{ }^{* * * *} \mathrm{P}<0.001\right)$. (C) Protein levels of ADAMTS1 prior to and following treatment with different concentrations of 5-Aza-dC, detected using immunofuorescence (magnification, x200). The MGC-803 cells immunostained with antibody to ADAMTS1 (green) were characterized by perinuclear cytoplasmic immunofluorescence. The cell nuclei were visualized with Hoechst33258 (blue). (D) Representative figures of MGC-803 cell invasion in untreated and 5-Aza-dC-treated MGC-803 cells. (a) Invasion in the untreated group; (b) invasion in the 5-Aza-dC-treated group. (E) Numbers of MGC-803 cells following treatment with 5-Aza-dC compared with the untreated control group. The values are presented as the mean \pm standard deviation (Mann-Whitney U test; $\mathrm{P}<0.001$ ). ADAMTS1, A disintegrin and metallopeptidase with thrombospondin motif type 1; 5-Aza-dC, 5-Aza-2'-deoxycytidine.

cancer, prostate cancer, lung cancer, pancreatic cancer and colorectal cancer $(18-20,25,27,28)$. These findings are in accordance with our previous study, in which the expression of ADAMTS1 was frequently reduced in primary gastric cancer (22). In the present study, the mRNA expression of ADAMTS1 was significantly lower in the gastric cancer cell lines compared with the normal gastric cell line. Together, these findings support the hypothesis that ADAMTS1 functions as a tumor inhibitor in the gastric cancer. Inversely,
ADAMTS1 protein can exert pro- and anti-angiogenic properties, dependent upon its proteolytic status (29). Several reports have revealed that the expression of ADAMTS1 was elevated in endometrial adenocarcinoma (10) and breast cancer (30). Metastatic cancer was often associated with increased ADAMTS1 (29,31). These findings suggest the binary role of ADAMTS1 in cancer.

It has been reported that the expression of ADAMTS1 is epigenetically silenced in non-small cell lung cancer 

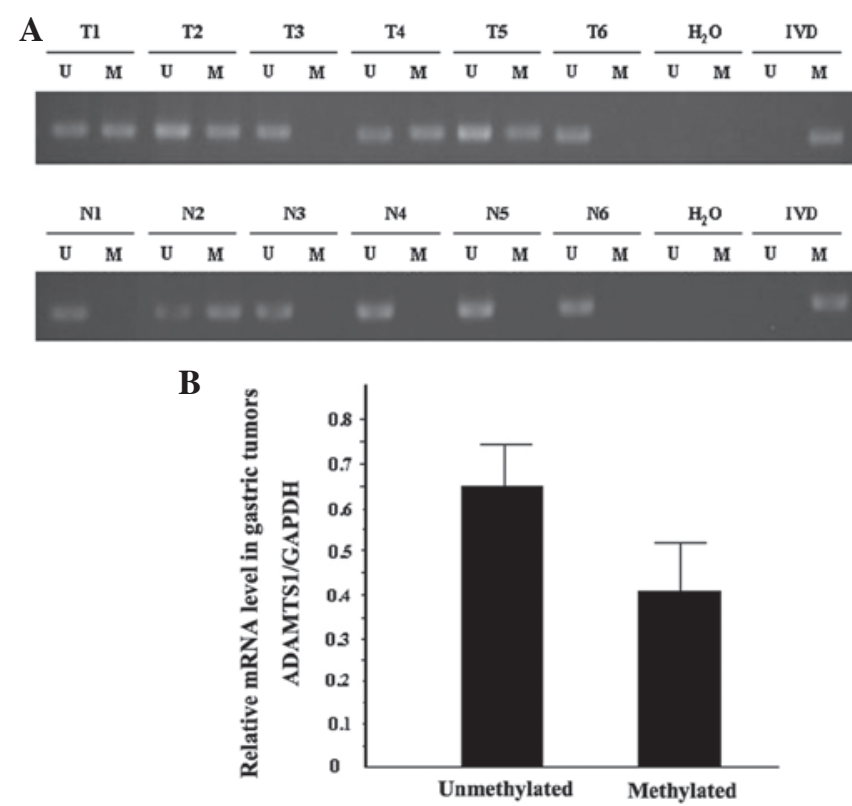

Figure 5. Methylation analysis of ADAMTS1 in gastric tissue samples. (A) Methylation of ADAMTS1 in primary gastric tumor tissues and corresponding normal gastric tissues. (B) Relative mRNA levels of ADAMTS1 in the unmethylated primary gastric tumor tissues and methylated primary gastric tumor tissues (Mann-Whitney U test; $\mathrm{P}=0.043$ ). Data are presented as the mean \pm standard deviation. $\mathrm{N}$, normal tissue; $\mathrm{T}$, tumor tissue; $\mathrm{U}$, unmethylated; $\mathrm{M}$, methylated; IVD, positive control; $\mathrm{H}_{2} \mathrm{O}$, negative control. ADAMTS1, A disintegrin and metallopeptidase with thrombospondin motif type 1 .

(NSCLC) $(25,32)$, prostate cancer (33) and colorectal tumor tissues $(28,34)$, however, no significant association has been observed between hypermethylation and reduced expression of ADAMTS1 in colorectal tumor tissues (34). Methylation in NSCLC tumor tissues is not correlated with clinicopathologic features of the patients, including age, gender, histology and pathologic staging (25). In addition, several studies have revealed that the ADAMTS9 and ADAMTS18 gene promoters are also hypermethylated in gastric carcinoma $(35,36)$. There are no previous studies describing the methylation status of ADAMTS1 in human gastric tumor tissues. The present study demonstrated that the frequency of ADAMTS1 methylation in primary gastric tumors and gastric cancer cell lines was significantly higher than in normal gastric tissues and the normal gastric cell line. In addition, the downregulation in the expression of ADAMTS1 was correlated with methylation of its promoter in the gastric cancer cell lines and gastric cancer tissues. The results also revealed that tumors with an invasion depth at T3 and T4 had markedly higher methylation frequencies than those with an invasion depth at $\mathrm{T} 1$ and $\mathrm{T} 2$. The methylation frequency of ADAMTS1 was also negatively associated with TNM stage. These results suggested that aberrant methylation of ADAMTS1 was involved in the pathogenesis of gastric cancer, and the invasion ability of gastric cancer may be higher when the methylation frequency of ADAMTS1 is high.

5-Aza-dC, a demethylating agent, is a nucleoside analog, which can inhibit DNA cytosine methylation, including reactivation of epigenetically silenced genes (37). In the present study 5-Aza-dC reactivated the expression of ADAMTS1. This suggested that reactivation of silenced genes correlated with decreased DNA methylation. The effect of 5-Aza-dC treatment on cancer cell invasion was determined using a Matrigel invasion assay. The number of invading MGC-803 cells was significantly reduced following treatment with 5 -Aza-dC, which indicated that 5-Aza-dC inhibited the invasive ability of the MGC-803 cells. These results revealed that DNA demethylation decreased the invasive force of the gastric cancer cells. An understanding of the precise mechanism of the down-=regulation of ADAMTS1 in gastric cancer needs requires further investigation.

In conclusion, the results of the present study demonstrated that the mRNA expression of ADAMTS1 was significantly lower in gastric cancer cell lines. Furthermore, the frequency of ADAMTS1 methylation was significantly higher in primary gastric tumor tissues than in gastric cancer cell lines. The downregulation in the expression of ADAMTS1 was correlated with its promoter methylation in the primary gastric tumors and gastric cancer cell lines. These results suggested that the anti-angiogenic function of ADAMTS1 is primarily important in primary gastric cancer. This function is lost by epigenetic silencing in tumor cells and tumor tissues. Aberrant methylation may be involved in the development and progression of gastric cancer.

\section{Acknowledgements}

This study was supported by a grant from the Natural Science Foundation of Liaoning Province (no. 2013021033), the Higher Specialized Research Fund from the Doctoral Program of the Ministry of Education of China (no. 20102104110001) and the Liaoning Province Science and Technology Plan Project (no. 2013225021).

\section{References}

1. Porter S, Clark IM, Kevorkian L and Edwards DR: The ADAMTS metalloproteinases. Biochem J 386: 15-27, 2005.

2. Tang BL and Hong W: ADAMTS: a novel family of proteases with an ADAM protease domain and thrombospondin 1 repeats. FEBS Lett 445: 223-225, 1999.

3. Kaushal GP and Shah SV: The new kids on the block: ADAMTSs, potentially multifunctional metalloproteinases of the ADAM family. J Clin Invest 105: 1335-1337, 2000.

4. Colige A, Sieron AL, Li SW, et al: Human Ehlers-Danlos syndrome type VII C and bovine dermatosparaxis are caused by mutations in the procollagen I N-proteinase gene. Am J Hum Genet 65: 308-317, 1999.

5. Levy GG, Nichols WC, Lian EC, et al: Mutations in a member of the ADAMTS gene family cause thrombotic thrombocytopenic purpura. Nature 413: 488-494, 2001.

6. Dunn JR, Panutsopulos D, Shaw MW, et al: METH-2 silencing and promoter hypermethylation in NSCLC. Br J Cancer 91: 1149-1154, 2004.

7. Glasson SS, Askew R, Sheppard B, et al: Characterization of and osteoarthritis susceptibility in ADAMTS-4-knockout mice. Arthritis Rheum 50: 2547-2558, 2004.

8. Porter S, Scott SD, Sassoon EM, et al: Dysregulated expression of adamalysin-thrombospondin genes in human breast carcinoma. Clin Cancer Res 10: 2429-2440, 2004.

9. Llamazares M, Obaya AJ, Moncada-Pazos A, et al: The ADAMTS12 metalloproteinase exhibits anti-tumorigenic properties through modulation of the Ras-dependent ERK signalling pathway. J Cell Sci 120: 3544-3552, 2007.

10. Keightley MC, Sales KJ and Jabbour HN: PGF2alpha-Fprostanoid receptor signalling via ADAMTS1 modulates epithelial cell invasion and endothelial cell function in endometrial cancer. BMC Cancer 10: 488, 2010.

11. Mochizuki S and Okada Y: ADAMs in cancer cell proliferation and progression. Cancer Sci 98: 621-628, 2007. 
12. Jones GC and Riley GP: ADAMTS proteinases: a multi-domain multi-functional family with roles in extracellular matrix turnover and arthritis. Arthritis Res Ther 7: 160-169, 2005.

13. Sandy JD, Westling J, Kenagy RD, et al: Versican V1 proteolysis in human aorta in vivo occurs at the Glu441-Ala442 bond, a site that is cleaved by recombinant ADAMTS-1 and ADAMTS-4. J Biol Chem 276: 13372-13378, 2001.

14. Russell DL, Doyle KM, Ochsner SA, Sandy JD and Richards JS: Processing and localization of ADAMTS-1 and proteolytic cleavage of versican during cumulus matrix expansion and ovulation. J Biol Chem 278: 42330-42339, 2003

15. Kuno K, Bannai K, Hakozaki M, Matsushima K and Hirose K: The carboxyl-terminal half region of ADAMTS-1 suppresses both tumorigenicity and experimental tumor metastatic potential Biochem Biophys Res Commun 319: 1327-1333, 2004.

16. Obika M, Ogawa H, Takahashi K, et al: Tumor growth inhibitory effect of ADAMTS1 is accompanied by the inhibition of tumor angiogenesis. Cancer Sci 103: 1889-1897, 2012.

17. Luque A, Carpizo DR and Iruela-Arispe ML: ADAMTS1/METH1 inhibits endothelial cell proliferation by direct binding and sequestration of VEGF165. J Biol Chem 278: 23656-23665, 2003

18. Gustavsson H, Wang W, Jennbacken K, Welen K and Damber JE: ADAMTS1, a putative anti-angiogenic factor, is decreased in human prostate cancer. BJU Int 104: 1786-1790, 2009.

19. Freitas VM, do AJB, Silva TA, et al: Decreased expression of ADAMTS-1 in human breast tumors stimulates migration and invasion. Mol Cancer 12: 2, 2013.

20. Martino-Echarri E, Fernandez-Rodriguez R, Rodriguez-Baen a FJ, et al: Contribution of ADAMTS1 as a tumor suppressor gene in human breast carcinoma. Linking its tumor inhibitory properties to its proteolytic activity on nidogen-1 and nidogen- 2 . Int J Cancer 133: 2315-2324, 2013.

21. Toyota M, Ahuja N, Suzuki H, et al: Aberrant methylation in gastric cancer associated with the $\mathrm{CpG}$ island methylator phenotype. Cancer Res 59: 5438-5442, 1999.

22. Chen J, Zhi Y, Chang X, Zhang S and Dai D: Expression of ADAMTS1 and its correlation with angiogenesis in primary gastric cancer and lymph node metastasis. Dig Dis Sci 58: 405-413, 2013.

23. Zhi Y, Chen J, Zhang S, Chang X, Ma J and Dai D: Down-regulation of CXCL12 by DNA hypermethylation and its involvement in gastric cancer metastatic progression. Dig Dis Sci 57: 650-659, 2012.

24. Herman JG, Graff JR, Myohanen S, Nelkin BD and Baylin SB: Methylation-specific PCR: a novel PCR assay for methylation status of CpG islands. Proc Natl Acad Sci USA 93 9821-9826, 1996.
25. Choi JE, Kim DS, Kim EJ, et al: Aberrant methylation of ADAMTS1 in non-small cell lung cancer. Cancer Genet Cytogenet 187: 80-84, 2008.

26. Lee NV, Sato M, Annis DS, et al: ADAMTS1 mediates the release of antiangiogenic polypeptides from TSP1 and 2. EMBO J 25: 5270-5283, 2006.

27. Masui T, Hosotani R, Tsuji S, et al: Expression of METH-1 and METH-2 in pancreatic cancer. Clin Cancer Res 7: 3437-3443, 2001.

28. Ahlquist T, Lind GE, Costa VL, et al: Gene methylation profiles of normal mucosa and benign and malignant colorectal tumors identify early onset markers. Mol Cancer 7: 94, 2008.

29. Liu YJ, Xu Y and Yu Q: Full-length ADAMTS-1 and the ADAMTS-1 fragments display pro- and antimetastatic activity, respectively. Oncogene 25: 2452-2467, 2006.

30. Tyan SW, Hsu CH, Peng KL, et al: Breast cancer cells induce stromal fibroblasts to secrete ADAMTS1 for cancer invasion through an epigenetic change. PLoS One 7: e35128, 2012.

31. Ricciardelli C, Frewin KM, Tan IA, et al: The ADAMTS1 protease gene is required for mammary tumor growth and metastasis. Am J Pathol 179: 3075-3085, 2011.

32. Kohno T, Otsuka A, Girard L, et al: A catalog of genes homozygously deleted in human lung cancer and the candidacy of PTPRD as a tumor suppressor gene. Genes Chromosomes Cancer 49: 342-352, 2010.

33. Yegnasubramanian S, Wu Z, Haffner MC, et al: Chromosomewide mapping of DNA methylation patterns in normal and malignant prostate cells reveals pervasive methylation of gene-associated and conserved intergenic sequences. BMC Genomics 12: 313, 2011.

34. Lind GE, Kleivi K, Meling GI, et al: ADAMTS1, CRABP1 and $\mathrm{NR} 3 \mathrm{C} 1$ identified as epigenetically deregulated genes in colorectal tumorigenesis. Cell Oncol 28: 259-272, 2006.

35. Li Z, Zhang W, Shao Y, et al: High-resolution melting analysis of ADAMTS18 methylation levels in gastric, colorectal and pancreatic cancers. Med Oncol 27: 998-1004, 2010.

36. Zhang C, Shao Y, Zhang W, et al: High-resolution melting analysis of ADAMTS9 methylation levels in gastric, colorectal and pancreatic cancers. Cancer Genet Cytogenet 196: 38-44, 2010.

37. Singh KP, Treas J, Tyagi T and Gao W: DNA demethylation by 5 -aza-2-deoxycytidine treatment abrogates 17 beta-estradiol-induced cell growth and restores expression of DNA repair genes in human breast cancer cells. Cancer Lett 316: 62-69, 2012. 